\title{
A Study on the Relationships Between Drivers' Emotions and Brain Signals
}

\author{
Songyi Chae ${ }^{(\bowtie)}$ \\ Human and Systems Engineering, UNIST, \\ 100 Banyeon-ri, Eonyang-eup, Ulju-gun, Ulsan, Korea \\ robim0314@gmail.com
}

\begin{abstract}
In this study, the correlation between six basic emotions (happy, sad, angry, disgusted, scared, and neutral) and brain signals evoked by head-up display (HUD) images were found. 20 participants were exposed to 18 different HUD images in the laboratory and driving simulator-based settings. 16-channel electroencephalography (EEG) signals were obtained during exposure to each HUD image and were later used to calculate three EEG indices (EEG activity, concentration level and relaxation level). The participants reported their emotions induced by the observation of each HUD image on semantic differential scales with two bipolar adjectives (range: 1-7). Results showed that color was a key factor for determining the type of emotion evoked, whereas the amount of information provided determined the levels of brain activity in the central lobe. Neural activities in the temporal lobe showed a strong association with the concentration level. These findings between emotions and EEG signals can be used for designing a new type of DVI (driver-vehicle interface).
\end{abstract}

Keywords: Driver's emotion · Brain signal · Electroencephalography (EEG) • Driver-vehicle interface (DVI)

\section{Introduction}

Driving is a task which requires the simultaneous processing of various visual stimuli [1]. A head-up display (HUD) interface is a device which minimizes eye movement of a driver and provides the driver with important information, such as the speed, fuel gauge and navigation, at the front of the vehicle [2]. Thus, HUD enables a driver to concentrate on the road conditions ahead by eliminating reasons for disengaging their vision from infront of the steering wheel. The HUD image is easily recognized, even by first-time drivers [3], and generally enables drivers to feel safer while driving [4].

An extensive amount of research about HUD hardware design has been conducted, however the number of studies which concentrate on HUD image design is insufficient. Various aspects such as the reaction and performance rates corresponding to the provided information from HUD should be considered in HUD image design [1]. HUD image design must be suited to evoke positive emotions of the driver as they heavily impact driving performance and furthermore, the safety of both the driver and other vehicles. Therefore, this study investigates the electroencephalogram (EEG) signals and the emotions evoked by HUD images of the driver in prior research conducted by Smith in 2011 [5]. 
In Smith's research, six pairs of emotion provoked by each of the 18 HUD images, were established by conducting a survey. This paper considers Smith's experiment as a foundation for HUD image design, hence uses existing HUD images to evaluate the emotions and EEG signals of drivers, therefore establishing a correlation between the two properties. Furthermore, the paper aims to develop a driver-vehicle interface (DVI) which investigates the elements of HUD image design that affect the emotions and EEG signals of drivers.

\section{Method}

\subsection{Overview of Experiment}

20 participants in both laboratory and driving-simulator based settings were exposed to visual stimuli in order to investigate the emotions and brain signals evoked by 18 HUD images (see Fig. 1) of various design elements including; content, amount of information, location, font, and color. 16 channels of EEG signals were measured while the participants observed each HUD image, in order to calculate the three EEG indices of; EEG activity, concentration level and relaxation level. Participants rated on scale of 1 to 7 , the amount of emotion induced by the HUD images in regards to five pairs of antonyms and six individual emotive adjectives.

\subsection{Participants}

In this experiment, 20 participants with at least one year of driving experience were involved, consisting of 10 males and 10 females aged between 20 and 31 years of age. All participants did not have any auditory, physical or mental disorders.

\subsection{Apparatus}

The 18 HUD images used in the experiment are displayed in Fig. 1, while their characteristics are listed in Table 1. Each image was a different combination of 6 design elements including; form of main information, form of sub information, the amount of information, location, font, and color. Each design element had sub design level, where colour has 3 levels; orange, blue and green.

Poly G-A (Laxtha. Korea) equipment was used to investigate the brain waves of each participant and a sampling frequency of $256 \mathrm{~Hz}$ was used to produce EEG signals. The EEG electrodes used 16 channels according to the International 10-20 System of Electrode Placement and the attaching positions were Fp1, Fp2, F3, F4, F7, F8, C3, C4, T7, T8, P3, P4, P7, P8, O1, O2, as shown in Fig. 2.

\subsection{Experimental Environment}

Experiments were conducted under laboratory-based and driving simulator-based settings (see Fig. 3). In the laboratory, a basic experiment was conducted using the the 


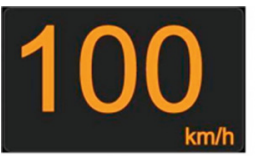

1

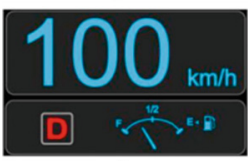

4

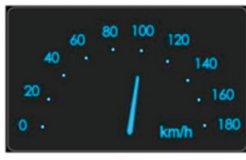

7

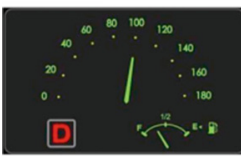

10

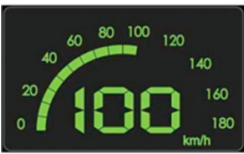

13

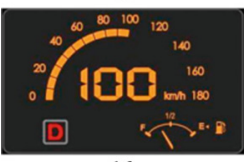

16

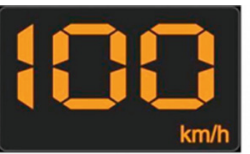

2
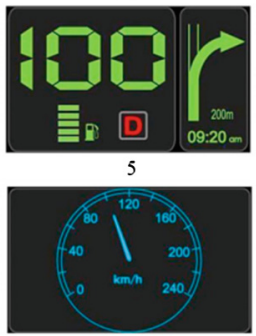

8

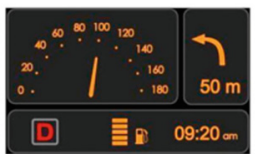

11

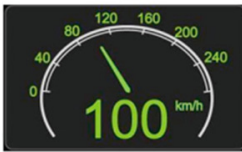

14

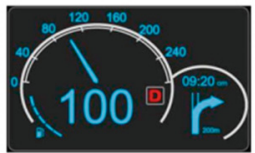

17
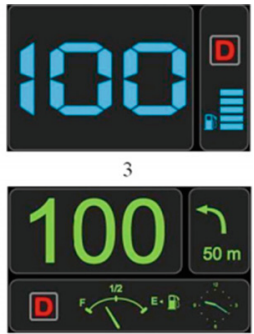

6
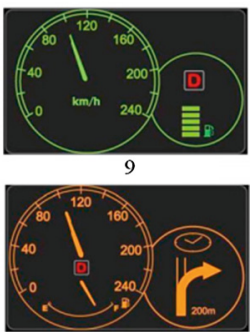

12

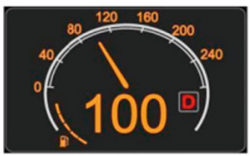

15

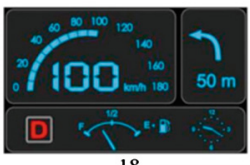

18

Fig. 1. 18 kinds of HUD images [5] (Smith, 2011) (Color figure online)

Table 1. Design factors of HUD images [5] (Smith, 2011)

\begin{tabular}{l|l|l|l}
\hline Design elements & & Sub design elements & \\
\hline Main information form & Digital & Meter & Mixture \\
\hline Sub information form & Digital & Meter & \\
\hline Amount of information & 1 item & 3 items & 5 items \\
\hline & Speedometer & Speedometer & Speedometer \\
\hline & & Position of gear & Position of gear \\
\hline & & Fuel gauge & Fuel gauge \\
\hline & & & Clock \\
\hline Location & & & Navigation \\
\hline Font & Left & Center & Right \\
\hline Color & Arial & Electronic & \\
\hline & Orange & Green & Blue \\
\hline
\end{tabular}




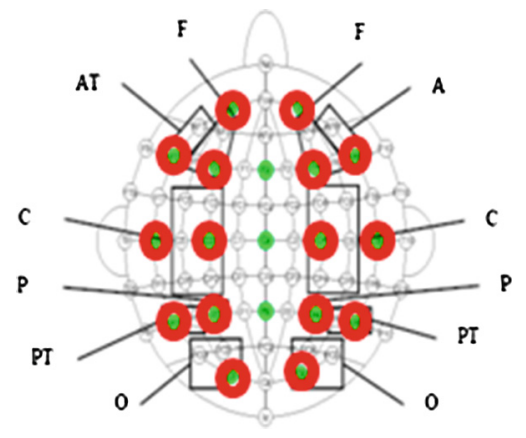

Fig. 2. Positions of EEG electrode attachments (Color figure online)

Geneva Affective PicturE Database (GAPED) in order to classify the possible emotions experienced by participants, before undertaking the HUD image experiment. The GAPED consists of six categories of visual emotion stimuli, each inducing an emotion of; scared, disgusted, angry, sad, neutral and happy. After the basic experiment, the 18 HUD images of $180 \mathrm{~mm} \times 110 \mathrm{~mm}$ were displayed on a laptop.
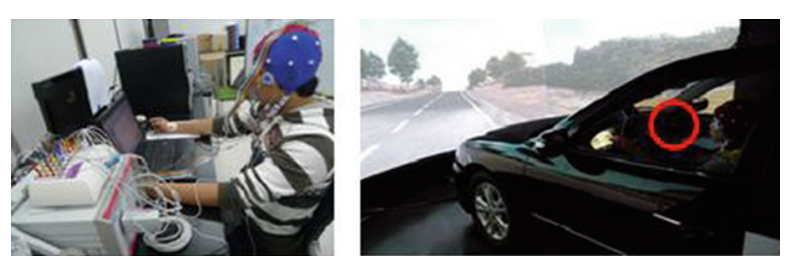

Fig. 3. Laboratory (left) and driving simulator (right) environments

In the driving simulator-based experiment, the same HUD images were displayed on the right side of the center fascia.

The 16-channel EEG signals were measured while the participants observed the HUD images, in order to calculate the three EEG indices which measure the levels of EEG activity, concentration and relaxation of each participant. After observing each image, five pairs of antonyms and six individual emotive adjectives were rated on a scale of 1 to 7 , corresponding to the amount of each emotion evoked by the images.

The five pairs of antonyms were; classic-modern, masculine-feminine, comfortable-insecure, soft-rough, certain-uncertain. For example, a score of 1 regarding 'masculine-feminine' means that the image articulated strong masculinity. Similarly, a score of 4 for 'comfortable-insecure' would suggest that the image conveyed neither a comfortable or insecure feeling to the participant, however the impression of the image was closer to being insecure. The six individual emotive adjectives were catagorized as; scared, disgusted, angry, sad, neutral and happy. 


\subsection{Experimental Procedure}

The experiment was conducted for two hours. Participants closed their eyes for $30 \mathrm{~s}$ before being exposed to a HUD image for $30 \mathrm{~s}$. The brain signals emitted during the observation of the image were recorded and evaluated. After the observation of the image, a survey was completed, regarding the emotions evoked by the image. This method of observing the image and answering questions regarding the image was repeated until all of the 18 HUD images were evaluated by each participant. The experiment was then repeated, displaying the images in random order. Again, after viewing the image for $30 \mathrm{~s}$, each participant completed a survey until all of the 18 HUD images were evaluated twice, firstly in numerical order and then in a random order.

\subsection{Data Analysis}

The EEG signals corresponding to each emotion were evaluated after the experiment using the GAPED, which uses more drastic or explicit images as an effort to increase the availability of visual emotion stimuli. Noise in the recorded EEG signals was eliminated by pre-processing the data using frequencies in the range of 4 to $45 \mathrm{~Hz}$, using a fast Fourier transform band pass filter. The three EEG analysis indicators of EEG activity, concentration and relaxation levels were considered. Alpha waves increased when at a comfortable state, and beta waves showed an increase at states of intentional activity, insecurity and tension. These characteristics were used to derive the following:

$$
\text { EEG activity }=\text { Power Ratio of (Beta/Alpha) }
$$

Theta waves decreased in the concentration state. The concentration level was defined by the SMR rhythm $(12-15 \mathrm{~Hz})$ which appeared during the unfocused attention state and the mid-beta rhythm $(16-20 \mathrm{~Hz})$ which appeared during the focused attention state:

$$
\text { Concentration level }=\text { Power Ratio of }(\mathrm{SMR}+\mathrm{Mid}-\text { Beta }) / \text { Theta }
$$

The relaxation level was observed at a relaxed state, and was calculated as the ratio of Alpha Power to High-Beta power.

$$
\text { Relaxation level }=\text { Power Ratio of }(\text { Alpha/High }- \text { Beta })
$$

\section{Results}

\subsection{Correlation Between Basic Emotions and EEG Signals}

The mean values and standard deviation of the EEG analysis indices were obtained for each emotion and are represented by Table 2. The EEG signals were measured in microvolts $(\mathrm{mV})$ however all values in Table 2 are represented as indices, therefore are unitless. 
Table 2. Mean indices of EEG analysis for each basic emotion

\begin{tabular}{l|l|l|l|l|l|l}
\hline & Scared & Disgusted & Sad & Angry & Neutral & Happy \\
\hline EEG Activity & $2.156 \pm 0.902$ & $1.938 \pm 0.336$ & $2.463 \pm 1.037$ & $1.366 \pm 1.081$ & $2.003 \pm 0.801$ & $2.380 \pm 1.005$ \\
\hline Concentration & $0.486 \pm 0.252$ & $1.940 \pm 0.922$ & $0.413 \pm 0.153$ & $0.280 \pm 0.220$ & $0.452 \pm 0.267$ & $0.500 \pm 0.276$ \\
\hline Relaxation & $1.913 \pm 0.689$ & $1.357 \pm 0.172$ & $1.708 \pm 0.820$ & $2.441 \pm 0.585$ & $1.576 \pm 0.354$ & $2.058 \pm 0.653$ \\
\hline
\end{tabular}

According to the frequency range of the EEG signals which affect the emotions stimulated by the HUD images, delta $(0-4 \mathrm{~Hz})$, theta $(0-4 \mathrm{~Hz})$, alpha $(8-13 \mathrm{~Hz})$, beta $(13-25 \mathrm{~Hz})$, and gamma $(25-30 \mathrm{~Hz})$ waves were investigated, along with the levels of EEG activity, concentration and relaxation. The four primary elements were extracted by principal component analysis, using the VariMax rotation method which involved Kaiser normalization. The factor transform matrix is represented by Table 3 and the factors of rotation space was obtained (see Fig. 4).

Table 3. Factor transformation matrix of emotion and EEG analysis indices

\begin{tabular}{l|r|l|l|l}
\hline & \multicolumn{1}{|l|}{1} & 2 & 3 & 4 \\
\hline Happy & -.875 & & & \\
\hline Disgusted & .824 & & & \\
\hline Angry & .786 & & & \\
\hline Neutral & -.632 & & & \\
\hline Scared & .542 & & & \\
\hline EEG activity & & -.943 & & \\
\hline Alpha wave & & .865 & & \\
\hline Relaxation & & .836 & & \\
\hline Beta wave & & & .932 & \\
\hline Concentration & & & .902 & \\
\hline Sad & & & & .922 \\
\hline
\end{tabular}

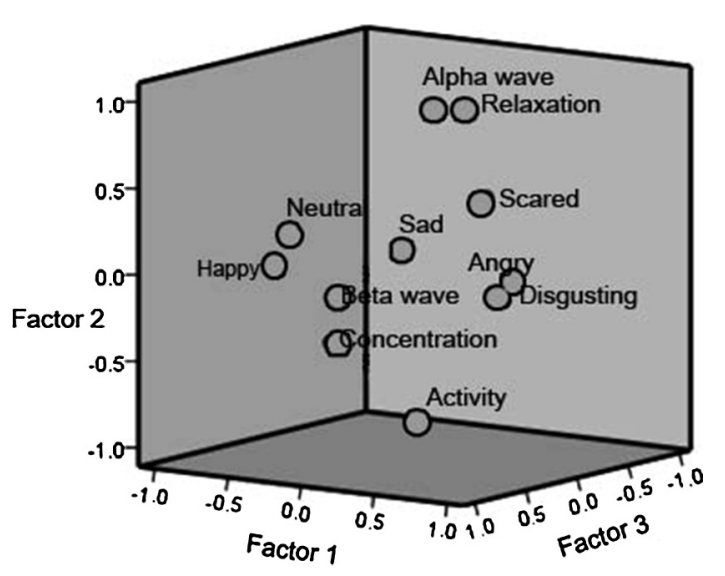

Fig. 4. Factors of emotion and EEG analysis indices 
The principal component analysis graph shown by Fig. 4 represents each factor as an axis in three-dimensional space and illustrates the correlations between the alpha and beta waves; emotions and levels of EEG activity, concentration and relaxation; experienced by participants during the HUD image experiment. Factor 1 consists of happy and neutral emotions, substantial concentration levels and beta waves, hence group positive elements. Factor 2 shows the presence of significant alpha waves and relaxation levels and also, disgusted, sad and angry emotions, indicating a general provocation of anxious and negative emotions. Factor 3 showed a weak correlation between the EEG activity and indices and the emotions induced by HUD images.

\subsection{Correlations Between HUD Images, Emotion and EEG Signals}

Correlation analysis was performed to investigate the correlation between emotion and EEG analysis indices while participants were exposed to HUD images.

Pearson's correlation coefficients and the results of pairwise statistical significance were obtained and are shown in Table 4.

Table 4. Correlation coefficients of emotion and EEG analysis indices

\begin{tabular}{|c|c|c|c|c|c|c|c|c|}
\hline & Activity & Concentration & Relaxation & Delta & Theta & Alpha & Beta & Gamma \\
\hline \multirow[t]{2}{*}{ Scared } & $-.657^{* * *}$ & .279 & .020 & $-.649^{* * *}$ & $.517^{* *}$ & $.678^{* * *}$ & $.446^{* * *}$ & $-.379^{*}$ \\
\hline & .000 & .100 & .907 & .000 & .001 & .000 & .006 & .023 \\
\hline \multirow[t]{2}{*}{ Disgusted } & $-.398^{*}$ & .043 & .315 & $-.598^{* * *}$ & .215 & $.464^{* * *}$ & $.568^{* * *}$ & -.023 \\
\hline & .016 & .803 & .061 & .000 & .208 & .004 & .000 & .893 \\
\hline \multirow[t]{2}{*}{ Sad } & $-.347^{*}$ & .038 & $.370^{*}$ & $-.659^{* *}$ & .215 & $.516^{* * *}$ & $.605^{* * *}$ & .029 \\
\hline & .038 & .825 & .026 & .000 & .207 & .001 & .000 & .867 \\
\hline \multirow[t]{2}{*}{ Angry } & $-.510^{* *}$ & .214 & .248 & $-.657^{* *}$ & $.351^{*}$ & $.579^{* *}$ & $.478^{* *}$ & -.119 \\
\hline & .001 & .209 & .145 & .000 & .036 & .000 & .003 & .491 \\
\hline \multirow[t]{2}{*}{ Neutral } & $.510^{* * *}$ & -.184 & -.231 & $.679^{* * *}$ & $-.399^{*}$ & $-.609^{* * *}$ & $-.510^{* * *}$ & .171 \\
\hline & .001 & .283 & .175 & .000 & .016 & .000 & .001 & .320 \\
\hline \multirow[t]{2}{*}{ Happy } & $-.362^{*}$ & .221 & $.435^{* *}$ & $-.667^{* * *}$ & .228 & $.546^{* * *}$ & $.574^{* * *}$ & .026 \\
\hline & .030 & .194 & .008 & .000 & .180 & .001 & .000 & .880 \\
\hline
\end{tabular}

Note: **. Correlation coefficient significance level: 0.01 (both sides), *. Correlation coefficient significance level: 0.01 (both sides)

Scared, disgusted, sad and angry were relevant to negative emotions, and they were of lower levels when EEG activity and delta waves increased at the significance level of 0.01 , however showed an increase when alpha and beta waves increased. For strong levels of happiness, which is a positive emotion, a strong correlation with the relaxation level, alpha and beta waves was shown when the significance level was 0.01 . Neutral emotion was experienced predominantly by the participants when EEG activity increased and theta beta waves were of lower levels.

The EEG activity in each part of the brain was different, depending on the nature of each HUD image and Fig. 5 indicates that for all 18 images, participants showed activity in the left frontal lobe while being exposed to visual stimuli. 


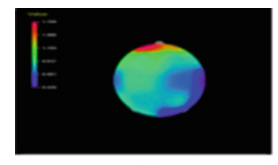

1
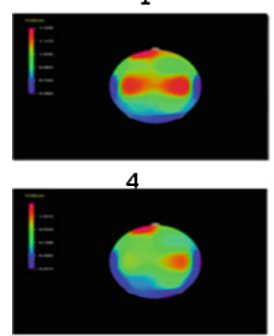

7

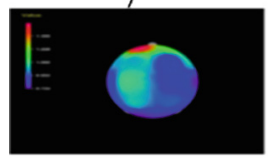

10

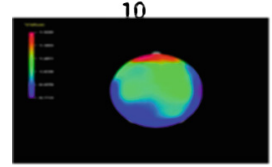

13

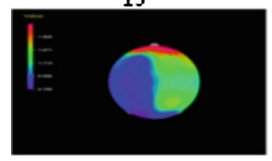

16

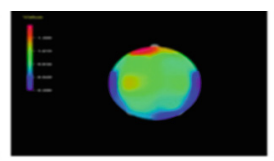

2

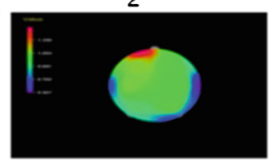

5

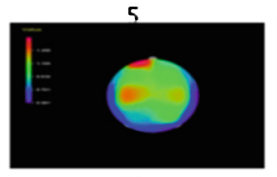

8

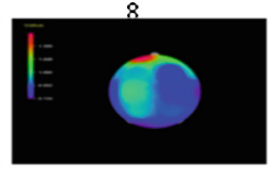

11

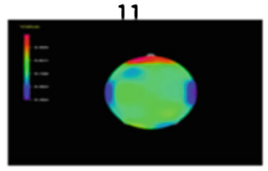

14

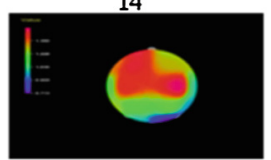

17

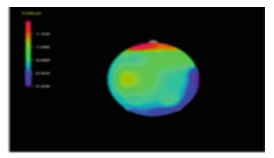

3

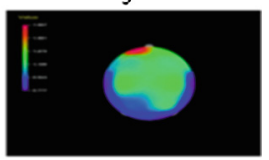

6

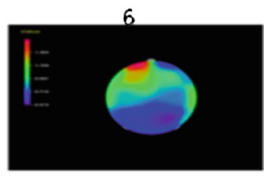

9

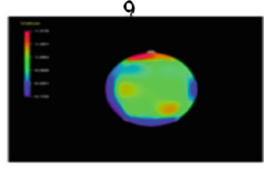

12

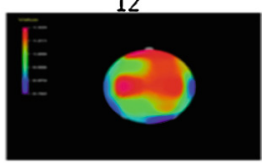

15

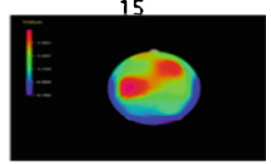

18

Fig. 5. EEG activity induced by each HUD image (Color figure online)

In the cases of the 4th, 15th, 7th and 18th HUD images, the parietal lobe was activated. One of the design elements of the image was the font, where Arial was used in every image except for the 18th image. Drivers experienced feelings of insecurity when the image was orange.

\subsection{Comparison of Results of Prior and Current HUD Image Research}

This study showed that emotions are induced by factors such as the amount and form of information provided, in which results of prior research were similar in a large percentage of cases. The results of the study showed a $80 \%$ compliance with that of Smith's experiment for the antonym pair, 'modern-classic', $71 \%$ for 'comfortableinsecure', and $100 \%$ for 'certain-uncertainly', respectively. The emotions evoked by both the colour and location of information corresponded to the adjective pairs 'masculine-feminine' and 'soft-rough' both showed a $55 \%$ in results of both studies, suggesting less compatibility between the current experiment and Smith's study. 


\section{Conclusion}

This research was conducted as an effort to improve the interface of a vehicle by grasping the difference between evoked emotions and EEG signals induced by HUD images, experienced by drivers. Before the HUD image experiment was conducted, the activated parts of the brain depending on the different kinds of emotion felt by the participant, were identified through a basic experiment.

Neutral emotions showed theta and alpha waves in the frontal and left temporal lobes. When participants experienced happiness, the presence of both delta waves in the frontal lobe and alpha waves showed differences in both right and left temporal lobes. Negative emotions such as feeling disgusted, angry or sad, indicated differences in the beta waves between the frontal and temporal lobes.

Through the HUD image experiment, a correlation between the emotions evoked by the images was able to be found, by considering the beta waves and concentration levels which are affected by positive and neutral emotions. Also, in the case of increased negative emotions, the relation between alpha waves and relaxation levels were noted. EEG activity had a weak correlation with emotions and other indices. EEG activity was proportional to the amount of information provided by the HUD and it showed significant differences at the parietal and temporal lobes. After the interpretation of short-answer responses, the most effective design element of HUD was concluded to be color, as it had dominant effects on the emotion of drivers.

EEG signals transmitted by observing HUD images used previously in Smith's experiment were analysed according to design elements and emotions as first step to identify the emotions induced visual stimuli, which may be experienced by drivers inside a vehicle. The study was limited by the fact that only 6 basic emotions were evaluated, instead of considering every emotion encountered by drivers inside a vehicle. Therefore, further research will be conducted in order to clearly identify the difference in EEG signals depending on the emotions experienced by the participant, through undertaking more specific short-answer surveys and various analysis methods.

\section{References}

1. Yoo, H., Tsimhoni, O., Green, P.: The effect of HUD warning location on driver responses. In: International Transportation Systems World Congress, pp. 1-10 (1999)

2. Wierwille, W.W: Development of an initial model relating driver in-vehicle visual demands to accident rate. In: Third Annual Mid-Atlantic Human Factors Conference Proceedings, Virginia Polytechnic Institute and State University, Blacksburg, VA (1995)

3. Liu, Y.C.: Effect of using head-up display in automobile context on attention demand and driving performance. Displays 24, 157-165 (2003)

4. Tonnis, M., Lange, C., Klinker, G.: Visual longitudinal and lateral driving assistance in the head-up display of cars. In: Proceedings of the Sixth IEEE and ACM International Symposium on Mixed and Augmented Reality, Nara, Japan, pp. 128-131 (2007)

5. Smith, S., Fu, S.H.: The relationships between automobile head-up display presentation images and drivers' Kansei. Displays 32, 58-68 (2011) 